\title{
Mei Symmetry of Time-Scales Euler-Lagrange Equations and Its Relation to Noether Symmetry
}

\author{
X.H. ZHAI ${ }^{a}$ AND Y. ZHANG,* \\ ${ }^{a}$ School of Science, Nanjing University of Science and Technology, Nanjing 210094, P.R. China \\ ${ }^{b}$ College of Civil Engineering, Suzhou University of Science and Technology, Suzhou 215011, P.R. China \\ (Received March 13, 2019; revised version April 23, 2019; in final form May 10, 2019)

\begin{abstract}
In this paper, the Mei symmetry of the Euler-Lagrange equations on time-scales and its relation to the Noether symmetry are investigated. The definition and criterion of Mei symmetry of the Lagrangian system on time-scales are given. The conditions and forms of new conserved quantities which are found from the Mei symmetry of the system are derived. In addition, the Noether symmetry of a variational problem for Lagrangian on time-scales under the action of infinitesimal generator vectors and its corresponding conserved quantity are discussed. The results show that the Euler-Lagrange equations on time-scales, the Noether identity and the Noether conserved quantity of the variational problem under discussion are the same with the criterion equations, the structural equation, and the conserved quantity of the Mei symmetry for the original Lagrangian system on time-scales, respectively. In the end, two examples are provided to illustrate applications of the results.
\end{abstract}

DOI: 10.12693/APhysPolA.136.439

PACS/topics: 11.10.Ef, 11.30.Na, 02.30.Hq

\section{Introduction}

The symmetry method plays an important role in finding conserved quantities of dynamical equations. The relationship between the symmetry and the conserved quantity was revealed firstly by Noether [1] in 1918. That is, a conserved quantity can be found from a symmetry. Except for the Noether symmetry method, the Lie symmetry method [2-4] and the Mei symmetry method [5] are the other two valuable methods in studying dynamical systems. The Lie symmetry is the invariance of differential equations of motion of systems under the infinitesimal transformations, while the Mei symmetry is the form invariance of differential equations of motion when dynamical functions are replaced by transformed functions under the infinitesimal transformations. Here, the dynamical functions are Lagrangian, Hamiltonian, Birkhoffian, non-potential generalized forces, holonomic and non-holonomic constraint forces, holonomic and nonholonomic constraint equations, non-conservative forces, etc. The three symmetries above can lead directly or indirectly to conserved quantities. The Noether conserved quantity, the Hojman conserved quantity [6], and the Mei conserved quantity are three main conserved quantities. There is also a close relationship between these symmetries. Many important results were obtained by Mei and other workers [7-14].

The calculus of time-scales, which has attracted much attention, was first proposed by Hilger [15] in order to unify continuous and discrete analysis. The theory of time-scales is really useful and plays an important role in modeling complex dynamical processes [16-18]. It not

*corresponding author; e-mail: weidiezh@gmail.com only reveals the discrepancies between continuous results and discrete results, but also helps avoid proving results twice. In 2004, Bohner and Hilscher developed the calculus of variations on time-scales [19]. Basic mathematical theories about this subject were fully developed in Refs. [20-28].

Moreover, applications of the Noether symmetry method and the Lie symmetry method have made great progress in control problems on time-scales, nonconservative systems on time-scales, Hamiltonian systems on time-scales, and Birkhoffian systems on timescales [29-34]. However, little research about the Mei symmetry of dynamical systems on time-scales has been done. It is also important to find its relations to the Noether symmetry and the Lie symmetry. In this paper, we will study the Mei symmetry for Lagrangian systems on time-scales. Its relation to the Noether symmetry is discussed from the point of view of a variational problem.

This paper is organized as follows. In Sect. 2, we give the definition and criterion of the Mei symmetry for the Lagrangian system on time-scales. Three kinds of conserved quantities led by the Mei symmetry of the system on time scales are given in Sect. 3. In Sect. 4, we provide a discussion about the relationships between the Mei symmetry and the Noether symmetry. Two examples are provided in Sect. 5 to show applications of the results. In the end, conclusions and future works are given.

\section{Mei symmetry of time-scales Euler-Lagrange equations}

For basic knowledge about the calculus on time-scales, the readers can refer to Refs. [16] and [17].

The equations of motion for Lagrangian system [19] on time-scales are 


$$
\frac{\Delta}{\Delta t} \frac{\partial L}{\partial q_{s}^{\Delta}}-\frac{\partial L}{\partial q_{s}^{\sigma}}=0,(s=1,2, \ldots, n),
$$

where the Lagrangian $L\left(t, q_{s}^{\sigma}(t), q_{s}^{\Delta}(t)\right): \mathbb{T} \times \mathbb{R}^{2 n} \rightarrow \mathbb{R}$ and the generalized coordinates $q_{s}(t): \mathbb{T} \rightarrow \mathbb{R}$ are assumed to be $C_{r d}^{1}$. Assuming that

$$
E_{s}=\frac{\Delta}{\Delta t} \frac{\partial}{\partial q_{s}^{\Delta}}-\frac{\partial}{\partial q_{s}^{\sigma}}
$$

Eq. (1) becomes

$$
E_{s}(L)=0,(s=1,2, \ldots, n) .
$$

Suppose

$$
\begin{aligned}
& t^{*}=T\left(t, q_{k}(t), \varepsilon\right)=t+\varepsilon \zeta\left(t, q_{k}(t)\right), \\
& q_{s}^{*}=Q_{s}\left(t, q_{k}(t), \varepsilon\right)=q_{s}(t)+\varepsilon \xi_{s}\left(t, q_{k}(t)\right) \\
& \quad(s, k=1,2, \ldots, n),
\end{aligned}
$$

are one-parameter infinitesimal transformations of group and let

$$
X=\zeta \frac{\partial}{\partial t}+\xi_{s} \frac{\partial}{\partial q_{s}}
$$

be the corresponding infinitesimal generator, where $\varepsilon \in \mathbb{R}$.

The Lagrangian $L$ becomes a new Lagrangian $L^{*}$ under the transformations (4) and (5), that is

$$
\begin{aligned}
& L^{*}=L\left(t^{*}, q_{s}^{* \sigma^{*}}, \frac{\Delta q_{s}^{*}}{\Delta t^{*}}\right)= \\
& \quad L\left(t+\varepsilon \zeta, q_{s}^{\sigma}+\varepsilon \xi_{s}^{\sigma}, \frac{q_{s}^{\Delta}+\varepsilon \xi_{s}^{\Delta}}{1+\varepsilon \Delta \zeta}\right),
\end{aligned}
$$

by performing Taylor series expansion at the point $\varepsilon=0$, we have

$$
L^{*}=L\left(t, q_{s}^{\sigma}, q_{s}^{\Delta}\right)+\varepsilon X^{(1)}(L)+O\left(\varepsilon^{2}\right),
$$

where

$$
X^{(1)}=\zeta \frac{\partial}{\partial t}+\xi_{s} \frac{\partial}{\partial q_{s}}+\left(\xi_{s}^{\Delta}-q_{s}^{\Delta} \zeta^{\Delta}\right) \frac{\partial}{\partial q_{s}^{\Delta}}
$$

is the first extended infinitesimal generator [34].

Definition 1. If the form of Eq. (3) stays invariant when the Lagrangian $L$ is replaced by the new Lagrangian $L^{*}$, i.e.

$$
E_{s}\left(L^{*}\right)=0, \quad(s=1,2, \ldots, n),
$$

then this invariance is called the Mei symmetry of equations of motion for the Lagrangian system on time-scales.

From Definition 1 and Eq. (8), we have

Criterion 1. If the infinitesimals $\zeta$ and $\xi_{s}$ satisfy

$$
E_{s}\left[X^{(1)}(L)\right]=0,
$$

then the corresponding invariance is the Mei symmetry for the Lagrangian system (3) on time-scales.

\section{New conserved quantities of Lagrangian systems on time-scales}

For the Lagrangian system (3) on time-scales, new conserved quantities can be led by the Mei symmetry.
Theorem 1. If the infinitesimals $\zeta$ and $\xi_{s}$ of the Mei symmetry of the system (3) and the gauge function $G_{1}\left(t, q_{s}^{\sigma}, q_{s}^{\Delta}\right)$ satisfy the following structural equation:

$$
\begin{aligned}
& X^{(1)}(L) \zeta^{\Delta}+X^{(1)}\left[X^{(1)}(L)\right] \\
& \quad+\mu(t) \frac{\partial X^{(1)}(L)}{\partial q_{s}^{\sigma}} q_{s}^{\Delta} \zeta^{\Delta}+G_{1}^{\Delta}=0,
\end{aligned}
$$

then the Mei symmetry of the system can lead to the new conserved quantity

$$
\begin{aligned}
I_{1} & =\frac{\partial X^{(1)}(L)}{\partial q_{s}^{\Delta}} \xi_{s}+\left[X^{(1)}(L)-\frac{\partial X^{(1)}(L)}{\partial q_{s}^{\Delta}} q_{s}^{\Delta}\right. \\
& \left.-\mu(t) \frac{\partial X^{(1)}(L)}{\partial t}\right] \zeta+G_{1}=\text { const. }
\end{aligned}
$$

Proof. To prove that Eq. (13) is a conserved quantity, we need to prove

$$
\frac{\Delta}{\Delta t} I=0 \text {. }
$$

More specifically, we have

$$
\begin{aligned}
& \frac{\Delta}{\Delta t} I_{1}=\frac{\partial X^{(1)}(L)}{\partial q_{s}^{\Delta}} \xi_{s}^{\Delta}+\frac{\Delta}{\Delta t}\left(\frac{\partial X^{(1)}(L)}{\partial q_{s}^{\Delta}}\right) \xi_{s}^{\sigma} \\
& +\left[X^{(1)}(L)-\frac{\partial X^{(1)}(L)}{\partial q_{s}^{\Delta}} q_{s}^{\Delta}-\mu(t) \frac{\partial X^{(1)}(L)}{\partial t}\right] \zeta^{\Delta} \\
& +\frac{\Delta}{\Delta t}\left[X^{(1)}(L)-\frac{\partial X^{(1)}(L)}{\partial q_{s}^{\Delta}} q_{s}^{\Delta}\right. \\
& \left.-\mu(t) \frac{\partial X^{(1)}(L)}{\partial t}\right] \zeta^{\sigma}+G_{1}^{\Delta} .
\end{aligned}
$$

Here, for the system (3), the following energy equation [26]:

$$
\frac{\Delta}{\Delta t}\left[L-\frac{\partial L}{\partial q_{s}^{\Delta}} q_{s}^{\Delta}-\mu(t) \frac{\partial L}{\partial t}\right]=\frac{\partial L}{\partial t}
$$

holds. After the transformation, the new energy equation

$$
\frac{\Delta}{\Delta t}\left[L^{*}-\frac{\partial L^{*}}{\partial q_{s}^{\Delta}} q_{s}^{\Delta}-\mu(t) \frac{\partial L^{*}}{\partial t}\right]=\frac{\partial L^{*}}{\partial t}
$$

holds by a similar proof of Eq. (16). Taking note of Eq. (8), we have

$$
\begin{aligned}
& \frac{\Delta}{\Delta t}\left[X^{(1)}(L)-\frac{\partial X^{(1)}(L)}{\partial q_{s}^{\Delta}} q_{s}^{\Delta}-\mu(t) \frac{\partial X^{(1)}(L)}{\partial t}\right]= \\
& \frac{\partial X^{(1)}(L)}{\partial t} .
\end{aligned}
$$

Thus, combining Eq. (12) with Eq. (18) yields

$$
\begin{aligned}
& \frac{\Delta}{\Delta t} I_{1}=X^{(1)}(L) \zeta^{\Delta}+X^{(1)}\left[X^{(1)}(L)\right] \\
& \quad+\mu(t) \frac{\partial X^{(1)}(L)}{\partial q_{s}^{\sigma}} q_{s}^{\Delta} \zeta^{\Delta}+G_{1}^{\Delta}=0 .
\end{aligned}
$$

The proof is completed. Theorem 1 gives the Mei conserved quantity (13) on time-scales led directly by the Mei symmetry of the system (3) while considering the structure equation (12). When $\mathbb{T}=\mathbb{R}$, the conserved quantity (13) becomes the classical Mei conserved quantity. 
Remark 1. If $\mathbb{T}=\mathbb{R}$, then $\sigma(t)=t, \mu(t)=0$, and the conserved quantity (13) becomes the classical one [5]:

$$
\begin{aligned}
I_{1} & =\frac{\partial X^{(1)}(L)}{\partial \dot{q}_{s}} \xi_{s} \\
& +\left[X^{(1)}(L)-\frac{\partial X^{(1)}(L)}{\partial \dot{q}_{s}} \dot{q}_{s}\right] \zeta+G_{1}=\text { const. }
\end{aligned}
$$

Remark 2. If $\mathbb{T}=h^{\mathbb{N}_{0}}=\left\{h^{i}: i \in \mathbb{N}_{0}\right\}$, where $h>1$ is a fixed real number, then $\sigma(t)=h t, \mu(t)=(h-1) t$, the conserved quantity (13) becomes the quantum one

$$
\begin{gathered}
I_{1}=\frac{\partial X^{(1)}(L)}{\partial q_{s}^{\Delta}} \xi_{s}+\left[X^{(1)}(L)-\frac{\partial X^{(1)}(L)}{\partial q_{s}^{\Delta}}\right. \\
\left.-(h-1) t \frac{\partial X^{(1)}(L)}{\partial t}\right] \zeta+G_{1}=\text { const },
\end{gathered}
$$

where $q_{s}^{\Delta}=\frac{q_{s}(h t)-q_{s}(t)}{(h-1) t}$.

Here, the calculus on $\mathbb{T}=h^{\mathbb{N}_{0}}=\left\{h^{i}: i \in \mathbb{N}_{0}\right\}, h>1$ is known as one type of quantum calculus [35], which helps to bridge the gap between the two families of models: continuous and discrete and is receiving an increased interest due to the applications in physics, economics, numerical analysis, and the calculus of variations [36-39].

Theorem 2. If the infinitesimals $\zeta$ and $\xi_{s}$ of the Mei symmetry of the system (3) and the gauge function $G_{2}\left(t, q_{s}^{\sigma}, q_{s}^{\Delta}\right)$ satisfy the following condition:

$$
q_{s}^{\sigma} \frac{\Delta}{\Delta t} \frac{\partial X^{(1)}(L)}{\partial q_{s}^{\sigma}}+q_{s}^{\Delta} \frac{\Delta}{\Delta t} \frac{\partial X^{(1)}(L)}{\partial q_{s}^{\Delta}}+G_{2}^{\Delta}=0,
$$

then the Mei symmetry of the system can lead to the new conserved quantity

$$
I_{2}=\frac{\partial X^{(1)}(L)}{\partial q_{s}^{\sigma}}\left[q_{s}^{\sigma}-\mu(t) q_{s}^{\Delta}\right]+G_{2}=\text { const. }
$$

By taking note of Eq. (11) and the condition (22), we can derive that Eq. (23) is a conserved quantity of the system (3).

Theorem 3. If the infinitesimals $\zeta$ and $\xi_{s}$ of the Mei symmetry of the system (3) and the gauge function $G_{3}\left(t, q_{s}^{\sigma}, q_{s}^{\Delta}\right)$ satisfy the following condition:

$$
\frac{\partial X^{(1)}(L)}{\partial t}+G_{3}^{\Delta}=0
$$

then the Mei symmetry of the system can lead to the new conserved quantity

$$
\begin{aligned}
I_{3} & =X^{(1)}(L)-\frac{\partial X^{(1)}(L)}{\partial q_{s}^{\Delta}} q_{s}^{\Delta} \\
& -\mu(t) \frac{\partial X^{(1)}(L)}{\partial t}+G_{3}=\text { const. }
\end{aligned}
$$

By taking note of Eqs. (18) and (24), we can derive that Eq. (25) is a conserved quantity of the system (3).

Theorem 2 and Theorem 3 give the other two kinds of conserved quantities (23) and (25) on time-scales also led by the Mei symmetry while considering the conditions (22) and (24).
The next section will show the relationship between the Mei symmetry and the Noether symmetry on the basis of Theorem 1.

\section{The relationship between Mei symmetry and Noether symmetry}

\subsection{The variational problem for Lagrangians on time-scales under the action of infinitesimal generator vector}

Let us consider integral functional $S[q(\cdot)]$ defined by:

$$
S[q(\cdot)]=\int_{a}^{b} X^{(1)}\left[L\left(t, q_{s}^{\sigma}(t), q_{s}^{\Delta}(t)\right)\right] \Delta t
$$

with the boundary condition

$$
\begin{aligned}
& \left.q_{s}(t)\right|_{t=a}=q_{s}(a),\left.\quad q_{s}(t)\right|_{t=b}=q_{s}(b), \\
& \quad(s=1,2, \cdots, n) .
\end{aligned}
$$

The necessary condition for an extremum of the functional (26) at $q_{s}=q_{s}(t)$ is that the variation of $S[q(\cdot)]$ is zero, i.e.

$\delta S[q(\cdot)]=\int_{a}^{b}\left(\frac{\partial X^{(1)}(L)}{\partial q_{s}^{\sigma}} \delta q_{s}^{\sigma}+\frac{\partial X^{(1)}(L)}{\partial q_{s}^{\Delta}} \delta q_{s}^{\Delta}\right) \Delta t=0$.

According to the formula of integration by parts of calculus on time-scales [16], we get

$$
\begin{aligned}
& \delta S[q(\cdot)]=\left.\left[\int_{a}^{t} \frac{\partial X^{(1)}(L)}{\partial q_{s}^{\sigma}} \Delta \tau \delta q_{s}\right]\right|_{a} ^{b} \\
& \quad+\int_{a}^{b}\left(\frac{\partial X^{(1)}(L)}{\partial q_{s}^{\Delta}}-\int_{a}^{t} \frac{\partial X^{(1)}(L)}{\partial q_{s}^{\sigma}} \Delta \tau\right)\left(\delta q_{s}\right)^{\Delta} \Delta t=0
\end{aligned}
$$

by applying the relations $\delta q_{s}^{\sigma}=\left(\delta q_{s}\right)^{\sigma}$ and $\delta q_{s}^{\Delta}=\left(\delta q_{s}\right)^{\Delta}$.

By considering the condition (27) and DuboisReymond lemma [19], we can obtain the Euler-Lagrange equation of the variational problem (26) and (27) with the same form of the criterion equation (11) of the Mei symmetry for the Lagrangian systems on time-scales (3).

\subsection{Noether symmetry of the variational problem (26) and (27)}

The Noether symmetry is about a kind of invariance of the action integral under infinitesimal transformations.

If the formula

$$
\begin{aligned}
& \int_{a}^{b} X^{(1)}\left[L\left(t, q_{s}^{\sigma}(t), q_{s}^{\Delta}(t)\right)\right] \Delta t= \\
& \int_{\alpha(a)}^{\alpha(b)} X^{(1)}\left[L\left(t^{*}, q_{s}^{\sigma^{*}}\left(t^{*}\right), q_{s}^{\Delta^{*}}\left(t^{*}\right)\right)\right] \Delta^{*} t^{*}
\end{aligned}
$$

holds under the transformations (4) and (5), then this invariance is called the Noether symmetry on time-scales. 
Here, the new time-scale map $t \rightarrow \alpha(t): T\left(t, q_{k}(t), \varepsilon\right)$ is assumed as an increasing $C_{r d}^{1}$ function and the symbol $\Delta^{*}$ and $\sigma^{*}$ denote the delta derivative and the forward jump operator, respectively. Also, we have the property $\sigma^{*} \circ \alpha=\alpha \circ \sigma$.

If the formula

$$
\begin{aligned}
& \int_{a}^{b} X^{(1)}\left[L\left(t, q_{s}^{\sigma}(t), q_{s}^{\Delta}(t)\right)\right] \Delta t= \\
& \quad \int_{\alpha(a)}^{\alpha(b)} X^{(1)}\left[L\left(t^{*}, q_{s}^{\sigma^{*}}\left(t^{*}\right), q_{s}^{\Delta^{*}}\left(t^{*}\right)\right)\right] \Delta^{*} t^{*} \\
& +\int_{a}^{b} \frac{\Delta}{\Delta t}\left(\hat{\Delta} G_{N}\right) \Delta t
\end{aligned}
$$

holds under the transformations (4) and (5), where $\hat{\Delta} G_{N}=\varepsilon G_{N}\left(t, q_{s}^{\sigma}, q_{s}^{\Delta}\right)$, then this invariance is called the Noether quasi-symmetry on time-scales. If $G_{N}=0$, this invariance is the Noether symmetry on time-scales.

From Eq. (31), for the infinitesimals $\zeta$ and $\xi_{s}$ there exists a gauge function $G_{N}\left(t, q_{s}^{\sigma}, q_{s}^{\Delta}\right)$ satisfying

$$
\begin{aligned}
& \frac{\partial X^{(1)}(L)}{\partial t} \zeta+\frac{\partial X^{(1)}(L)}{\partial q_{s}^{\sigma}} \xi_{s}^{\sigma}+\frac{\partial X^{(1)}(L)}{\partial q_{s}^{\Delta}}\left(\xi_{s}^{\Delta}-q_{s}^{\Delta} \zeta^{\Delta}\right) \\
& +X^{(1)}(L) \zeta^{\Delta}=-\frac{\Delta}{\Delta t} G_{N} .
\end{aligned}
$$

Formula (32) can be called the Noether identity of the variational problem (26) and (27). Note that the Noether identity (32) equals to the structural Eq. (12).

Theorem 4. For the variational problem (26) and $(27)$, if the infinitesimals $\zeta, \xi_{s}$ and the gauge function $G_{N}\left(t, q_{s}^{\sigma}, q_{s}^{\Delta}\right)$ satisfy the Noether identity (32), then the Noether quasi-symmetry can lead to the conserved quantity of the form (13) directly.

\section{Examples}

Example 1. The Lagrangian of a system on timescales with two degrees of freedom is

$$
L=\frac{1}{2}\left[\left(q_{1}^{\Delta}\right)^{2}+\left(q_{2}^{\Delta}\right)^{2}\right]-q_{1}^{\sigma} q_{2}^{\sigma}+t^{2} .
$$

Let us study the form invariance and the conserved quantity of the system.

The equations of the system are

$$
q_{2}^{\Delta \Delta}=-q_{1}^{\sigma}, q_{1}^{\Delta \Delta}=-q_{2}^{\sigma} .
$$

If we choose the first extended infinitesimal generator of the form

$$
X^{(1)}=q_{2} \frac{\partial}{\partial q_{1}}+q_{1} \frac{\partial}{\partial q_{2}}+q_{2}^{\Delta} \frac{\partial}{\partial q_{1}^{\Delta}}+q_{1}^{\Delta} \frac{\partial}{\partial q_{2}^{\Delta}},
$$

then the corresponding invariance is form invariance, because

$$
\begin{gathered}
X^{(1)}\left(\frac{1}{2}\left(q_{1}^{\Delta}\right)^{2}+\frac{1}{2}\left(q_{2}^{\Delta}\right)^{2}-q_{1}^{\sigma} q_{2}^{\sigma}+t^{2}\right)= \\
2 q_{1}^{\Delta} q_{2}^{\Delta}-\left(q_{1}^{\sigma}\right)^{2}-\left(q_{2}^{\sigma}\right)^{2}
\end{gathered}
$$

and

$$
E_{s}\left[2 q_{1}^{\Delta} q_{2}^{\Delta}-\left(q_{1}^{\sigma}\right)^{2}-\left(q_{2}^{\sigma}\right)^{2}\right]=0 .
$$

From Eq. (12), we have

$$
-4 q_{1}^{\sigma} q_{2}^{\sigma}+2 q_{2}^{\Delta} q_{2}^{\Delta}+2 q_{1}^{\Delta} q_{1}^{\Delta}=-\frac{\Delta}{\Delta t} G_{1} .
$$

Thus, we know that

$$
G_{1}=-2 q_{1} q_{1}^{\Delta}-2 q_{2} q_{2}^{\Delta}
$$

is a solution of (38). According to Theorem 1, the conserved quantity (13) of the system is

$$
I_{1}=0 \text {. }
$$

This conserved quantity is the trivial one.

According to Theorem 2, we obtain

$$
G_{2}=2 q_{1} q_{1}^{\sigma}+2 q_{2} q_{2}^{\sigma}
$$

and

$$
I_{2}=0 \text {. }
$$

This conserved quantity is also a trivial one.

According to Theorem 3, we obtain

$$
G_{3}=0
$$

and

$$
I_{3}=-\left(q_{1}^{\sigma}\right)^{2}-\left(q_{2}^{\sigma}\right)^{2}-2 q_{1}^{\Delta} q_{2}^{\Delta}=\text { const. }
$$

Conserved quantity (44) is led by form invariance of the system.

The results show that the Mei symmetry can lead to different conserved quantities under different conditions.

Example 2. The Lagrangian of a system on timescale $\mathbb{T}=h^{\mathbb{N}_{0}}=\left\{h^{i}: i \in \mathbb{N}_{0}\right\}$ is

$$
L=\frac{1}{2}\left[\left(q_{1}^{\Delta}(t)\right)^{2}+\left(q_{2}^{\Delta}(t)\right)^{2}\right]-q_{2}^{\sigma}(t),
$$

where $h>1$ is a constant and $\sigma(t)=h t, \mu(t)=$ $(h-1) t$.

The equations of the system are

$$
q_{1}^{\Delta \Delta}(t)=0, \quad q_{2}^{\Delta \Delta}(t)+1=0 .
$$

Considering the calculation, we have

$$
\begin{aligned}
& X^{(1)}(L)=-\xi_{2}^{\sigma}(t)+\left(\xi_{1}^{\Delta}(t)-q_{1}^{\Delta}(t) \zeta^{\Delta}(t)\right) q_{1}^{\Delta}(t) \\
& \quad+\left(\xi_{2}^{\Delta}(t)-q_{2}^{\Delta}(t) \zeta^{\Delta}(t)\right) q_{2}^{\Delta}(t) .
\end{aligned}
$$

If we choose the infinitesimals

$$
\zeta=\xi_{1}=0, \quad \xi_{2}^{\sigma}(t)=q_{2}^{\Delta}(t)+t,
$$

then we have

$$
\begin{aligned}
& E_{s}\left[X^{(1)}(L)\right]=E_{s}\left[-q_{2}^{\Delta}-t\right]=0, \quad(s=1,2), \\
& X^{(1)}\left[X^{(1)}(L)\right]=0 .
\end{aligned}
$$

Substituting (48) and (50) into (12), we obtain

$$
G_{1}=0
$$

and the conserved quantity (13) gives

$$
\begin{aligned}
& I_{1}=-q_{2}^{\Delta}(t-\mu(t))-(t-\mu(t))= \\
& \quad-q_{2}^{\Delta}((2-h) t)-(2-h) t=\mathrm{const} .
\end{aligned}
$$

From the discussion in Sect. 4, the integral functional (26) gives 


$$
\begin{gathered}
S=\int_{a}^{b} X^{(1)}\left[\frac{1}{2}\left(q_{1}^{\Delta}(t)\right)^{2}+\frac{1}{2}\left(q_{2}^{\Delta}(t)\right)^{2}-q_{2}^{\sigma}(t)\right] \Delta t= \\
\int_{a}^{b}\left(-q_{2}^{\Delta}-t\right) \Delta t
\end{gathered}
$$

with the boundary condition

$$
\left.q_{s}(t)\right|_{t=a}=q_{s}(a),\left.\quad q_{s}(t)\right|_{t=b}=q_{s}(b),(s=1,2) .
$$

Taking note of Theorem 4, we know that the infinitesimals (48) and Eq. (52) correspond to the Noether symmetry and Noether conserved quantity of the variational problem (53) and (54).

\section{Conclusions}

In recent years, the theory of time-scales calculus has become widely useful in describing complex dynamic process. The theory of symmetry is an important research aspect of analytical mechanics, mathematical physics, and dynamic control. This paper presents and studies the Mei symmetry for Lagrangian systems on time-scales. Three new forms of conserved quantities on time-scales are derived from the Mei symmetry of the system. In addition, the relation between the Mei symmetry and the Noether symmetry is discussed from the point of view of a variational problem for time-scales Lagrangian under the action of infinitesimal generator vector. The results of this paper cover not only the continuous results and the discrete results but also the results on time interval domain.

Further works about the Mei symmetry for nonconservative holonomic systems, non-holonomic systems, Hamiltonian systems, and Birkhoffian systems on time scales as well as its relations to Lie symmetry on timescales are still worth doing.

\section{Acknowledgments}

This work is supported by the National Natural Science Foundation of China (grant Nos. 11972241 and 11572212) and the Natural Science Foundation of Jiangsu Province (grant No. BK20191454).

\section{References}

[1] A.E. Noether, Nachr. Akad. Wiss. Gott. Math. Phys. 2, 235 (1918).

[2] M. Lutzky, J. Phys. A Math. Gen. 12, 973 (1979).

[3] M. Lutzky, Phys. Lett. A 72, 86 (1979).

[4] M. Lutzky, Phys. Lett. A 75, 8 (1979).

[5] F.X. Mei, J. Beijing Inst. Technol. 9, 120 (2000).

[6] S.A. Hojman, J. Phys. A Math. Gen. 25, L291 (1992).

[7] F.X. Mei, Applications of Lie Groups and Lie Algebras to Constrained Mechanical Systems, Science Press, Beijing 1999.
[8] F.X. Mei, Analytical Mechanics II, Beijing Institute Technology Press, Beijing 2013.

[9] J.F. Xie, S. K. Luo, L.Q. Jia, Chin. Phys. B 17, 1560 (2008).

[10] J.L. Cai, Acta Phys. Pol. A 115, 854 (2009).

[11] J.L. Cai, Acta Phys. Pol. A 117, 445 (2010).

[12] P. Wang, Y. Xue, Nonlin. Dyn. 83, 1815 (2016).

[13] W.A. Jiang, K. Liu, Z.W. Xia, M. Chen, Acta Mech. 229, 3781 (2018).

[14] S.K. Luo, M.J. Yang, X.T. Zhang, Y. Dai, Acta Mech. 229, 1833 (2018).

[15] S. Hilger, Ph.D. Thesis, Universtät Würzburg, 1988.

[16] M. Bohner, A. Peterson, Dynamic Equations on Time Scale: An Introduction with Applications, Birkhäuser, Boston 2001.

[17] M. Bohner, S.G. Georgiev, Multivariable Dynamic Calculus on Time Scales, Springer Int. Publ., Switzerland 2016.

[18] R.P. Agarwal, M. Bohner, D. O'Regan, A. Peterson, J. Comput. Appl. Math. 141, 1 (2002).

[19] M. Bohner, Dyn. Syst. Appl. 272, 339 (2004).

[20] R. ̌. Hilscher, V. Zeidan, J. Math. Anal. Appl. 289, 143 (2004).

[21] M. Bohner, S.G. Georgiev, Comput. Math. Appl. 54, 45 (2007).

[22] J. Cresson, A.B. Malinowska, D.F.M. Torres, Comput. Math. Appl. 64, 2294 (2012).

[23] R. Almeida, D.F.M. Torres, J. Vib. Control 15, 951 (2009).

[24] M. Dryl, D.F.M. Torres, Int. J. Differ. Eq. 8, 27 (2013).

[25] Z. Bartosiewicz, N. Martins, D.F.M. Torres, Eur. J. Control 17, 9 (2010).

[26] Z. Bartosiewicz, D.F.M. Torres, J. Math. Anal. Appl. 342, 1220 (2008).

[27] N. Martins, D.F.M. Torres, Appl. Math. Lett. 23 , $1432(2010)$

[28] Y. Zhang, J. Dyn. Control 38, 169 (2016).

[29] A.B. Malinowska, M.R.S. Ammi, Int. J. Differ. Eq. 9, 87 (2014).

[30] Y. Zhang, Chin. Q. Mech. 37, 214 (2016).

[31] C.J. Song, Y. Zhang, J. Math. Phys. 56, 102701 (2015).

[32] X.H. Zhai, Y. Zhang, Commun. Nonlin. Sci. Numer. Simul. 52, 32 (2017).

[33] P.P. Cai, J.L. Fu, Y.X. Guo, Rep. Math. Phys. 79, 279 (2017).

[34] X.H. Zhai, Y. Zhang, J. Vib. Control 25, 581 (2019).

[35] V. Kac, P. Cheung, Quantum Calculus, Springer, New York 2002.

[36] A.B. Malinowska, D.F.M. Torres, J. Optim. Theory Appl. 147, 419 (2010).

[37] K.A. Aldwoah, A.B. Malinowska, D.F.M. Torres, Dyn. Contin. Discrete Impuls. Syst. Ser. B Appl. Algorithms 19, 93 (2012).

[38] A.M.C. Brito da Cruz, N. Martins, D.F.M. Torres, Springer Proc. Math. Statist. 47, 359 (2013).

[39] J. Cresson, J. Math. Anal. Appl. 307, 48 (2005). 